\title{
CARIBEÑA DE
}

\section{CIENCIAS SOCIALES \\ latindex @iDEAs Econpapers oDialnet MIAR ÎnDICEs Surupira}

\section{SISTEMA DE MEDICIÓN DE LA CALIDAD DE LA EDUCACIÓN: ¿FINES PEDAGÓGICOS O RENDICIÓN DE CUENTAS?}

\author{
Bernardita Puentes Muñoz \\ Estudiante Doctorado en Educación \\ Universidad de Murcia \\ https://orcid.org/0000-0003-3806-0925 \\ bernarditacarmen.puentesm@um.es \\ María Luisa García Hernández \\ Profesora Contratada Doctor Permanente \\ Universidad de Murcia \\ https://orcid.org/0000-0002-1267-2571 \\ luisagarcia@um.es \\ Mónica Porto Currás \\ Profesora Titular de Universidad \\ Universidad de Murcia \\ https://orcid.org/0000-0002-4938-0572 \\ monicapc@um.es
}

Para citar este artículo puede utilizar el siguiente formato:

Bernardita Puentes Muñoz, María Luisa García Hernández y Mónica Porto Currás: "Sistema de medición de la calidad de la educación: ¿fines pedagógicos o rendición de cuentas?", Revista Caribeña de Ciencias Sociales (Especial noviembre 2021, pp. 74-87) En línea:

https://doi.org/10.51896/caribe/FTWA7354

\section{RESUMEN}

En Chile se ha instalado una cultura de la evaluación orientada fundamentalmente a la rendición de cuentas, que utiliza la prueba estandarizada SIMCE como mecanismo para medir los resultados de aprendizaje de los y las estudiantes y -con ello- la calidad del sistema. Sin embargo, a partir de la identificación de algunos efectos no deseados de la aplicación de esta prueba, la Agencia de Calidad de la Educación ha reorientado el uso de sus resultados desde un énfasis en el accountability a un foco centrado en la retroalimentación de la práctica pedagógica, con el fin de mejorar la calidad de la enseñanza. Enmarcado en este contexto, este estudio tuvo por objetivo general conocer la percepción del profesorado sobre el uso de los resultados de la prueba SIMCE en un centro de educación pública de Santiago de Chile, con el fin de averiguar si son utilizados desde esta nueva resignificación pedagógica. Para ello, se eligió un enfoque empírico-analítico, con un estudio descriptivo, utilizando un cuestionario elaborado ad hoc. Los datos muestran que coexiste un uso pedagógico de estos datos de aprendizaje en conjunto con aquellas acciones que deberían evitarse, por lo que el foco pedagógico no ha logrado desplazar la atención excesiva en la rendición de cuentas. 
Palabras clave: Calidad de la Educación, Medición, Rendición de cuentas, SIMCE, Práctica Pedagógica.

\section{EDUCATION QUALITY MEASUREMENT SYSTEM: PEDAGOGICAL PURPOSES OR ACCOUNTABILITY?}

\section{ABSTRACT}

In Chile, a mainly accountability-led culture of evaluation has been installed, which uses the SIMCE standardized test as a device to measure the learning outcomes of the students and -by doing so- the quality of the system. Nevertheless, in the light of the unwanted effects caused by the application of this test, the Agencia de Calidad de la Educación (The Quality Education Agency) has redirected the use of its results from an accountability-led approach to an approach more focused on teaching practice feedback, so as to improve the quality of teaching. From such framework, this research has, as a general objective, to know the perception of teachers about the use of the SIMCE test results in a public center of education in Santiago de Chile, aiming at finding out whether they are being used from this newly reset pedagogic scenario. With this purpose, an empiric-analytical approach was chosen, with descriptive research, using a questionnaire elaborated ad hoc. The data show that a pedagogical use of this learning data coexists alongside those actions that should be avoided, so that the pedagogical focus has failed to displace excessive attention on accountability.

Keywords: educational quality, measurement, accountability, SIMCE, teaching practice.

\section{INTRODUCCIÓN}

Lograr que los y las estudiantes puedan acceder a una educación de calidad es una de las metas que se han propuesto alcanzar los países de Hispanoamérica, pues su consecución puede impulsar el cambio social que permita superar la pobreza, mejorar la desigualdad en la distribución de los ingresos, incrementar la productividad y el desarrollo de la sociedad. Incluso se "interpreta la educación y la formación como las claves del progreso económico" (Ávila-Gómez, 2016, p. 59). De ahí que, según la Oficina Regional de Educación para América Latina y el Caribe (OREALC/UNESCO, 2013), el objetivo ha transitado desde aumentar únicamente la escolaridad a garantizar que el estudiantado efectivamente obtenga logros de aprendizaje.

Desde esta perspectiva, es posible desprender que la calidad es una exigencia de la sociedad actual y que en las últimas décadas ha encontrado en la evaluación el medio para conseguirla. La evaluación y la calidad tienen una relación estrecha siempre que la primera esté orientada a la mejora de los procesos de planificación, desarrollo y gestión educativa (García-Sanz, 2012). Asimismo, de acuerdo con la Organización de las Naciones Unidas para la Educación, la Ciencia y la Cultura (UNESCO, 2017), la evaluación de los aprendizajes es un elemento fundamental, pues recoge información sobre lo que saben los y las estudiantes y lo que pueden hacer con lo aprendido, además de aportar información sobre los procesos y los contextos que lo hacen posible y sobre los que pueden obstaculizarlo. 
Por ello, en lo referido a los logros alcanzados por el estudiantado, se observa en el mundo en general, y en Hispanoamérica en particular, un énfasis en la creación y aplicación de pruebas estandarizadas (OREALC/UNESCO, 2013; Díaz y Osuna, 2016), las que posibilitan estimar objetivamente el desempeño de enormes grupos de personas (Backhoff, 2018), razón por la que son empleadas como un instrumento para "medir el rendimiento de los alumnos, los docentes y los centros" (Moreno, 2016, p. 84). En esta línea, Aristizabal (2016) sostiene que una institución que dispone de una cultura para la gestión de datos académicos es una comunidad que crece, pues cuenta con espacios transparentes que posibilitan la discusión que lleva a la toma de decisiones de manera informada. Por lo tanto, se trata de una medición cuyos resultados sirven como base para aplicar acciones con alto impacto en los sistemas educativos de los países en los que se aplica (Rappoport y Sandoval, 2015) y - en particular- en el quehacer de las instituciones que imparten enseñanza (Moreno, 2016).

En relación con los efectos de la aplicación de estas pruebas, se reconocen tanto consecuencias positivas como negativas. Entre las primeras, es posible observar en el profesorado y en el equipo directivo una motivación adicional para mejorar los resultados de sus estudiantes (Anghel, Cabrales, Sainz y Sanz, 2013). Asimismo, "los países con sistemas de exámenes finales externos tienen un mejor comportamiento en las pruebas internacionales de rendimiento de los estudiantes" (Anghel et al., 2013, p. 2). En cuanto a sus efectos negativos, se identifican algunos nudos críticos, tales como el carácter descontextualizado de las pruebas o los desvíos que se generan en la enseñanza (Bordoli y Márquez, 2019).

Chile es un ejemplo de cómo la aplicación de una prueba estandarizada -denominada Sistema de Medición de la Calidad de la Educación (SIMCE)- ha creado e instalado una cultura de la evaluación basada fundamentalmente en el accountability o rendición de cuentas (Pino, Oyarzún y Salinas, 2016), pues se trata de una prueba asociada a la entrega de recursos y que propicia la competencia entre los centros (Carrasco y Urrejola, 2017). Ahora bien, una prueba que se ha utilizado principalmente para estos fines ha generado una serie de efectos no deseados entre los que se reconocen los siguientes: distorsión de las prácticas pedagógicas, homogeneización y estandarización de las prácticas de enseñanza, reducción curricular, agobio laboral y estrés en los y las docentes, entre otros (Botella y Ortiz, 2018).

En este contexto, la Agencia de Calidad de la Educación (organismo encargado de aplicar la prueba SIMCE en Chile) ha resignificado el propósito de esta evaluación, entregando orientaciones explícitas para que los resultados sean utilizados con fines pedagógicos (Agencia de Calidad de la Educación, 2018a). En consecuencia, se pretende que este instrumento de evaluación no se use exclusivamente para rendir cuentas, sino que sea valorado como una herramienta capaz de otorgar información que oriente las prácticas de los centros, con el fin de lograr aprendizajes en el estudiantado.

Para alcanzar este objetivo, la Agencia de Calidad de la Educación ha implementado mejoras 
en los Informes de Resultados Educativos de la prueba SIMCE con el propósito de facilitar la "comprensión y apropiación de la información por parte de las escuelas" (Agencia de Calidad de la Educación, 2018b, p. 19). En efecto, en este documento se presentan los resultados de aprendizaje desde un foco pedagógico, de tal forma que oriente el trabajo de los y las docentes, quienes podrán conocer las áreas específicas donde se encuentran las fortalezas y dificultades de sus estudiantes. Por lo tanto, se concibe a la evaluación como "una experiencia y una fuente continua de aprendizajes" (Moreno, 2017, p. 151) que favorecería el desempeño de los centros escolares.

Dado que es posible identificar en Chile una cultura de la evaluación a partir de la aplicación ininterrumpida de la prueba SIMCE desde hace más de treinta años y que la "herencia de la evaluación" (Moreno, 2011) consolida y arraiga ciertas prácticas en la comunidad educativa relacionadas fundamentalmente -en este caso- con la rendición de cuentas, surge la necesidad de investigar si efectivamente se ha instalado en la práctica pedagógica esta nueva resignificación impulsada por la Agencia de Calidad de la Educación. Por lo tanto, este estudio intenta averiguar si los y las docentes siguen las orientaciones de la Agencia sobre la utilización del Informe de Resultados Educativos de la prueba SIMCE desde una mirada pedagógica, evitando aquellas prácticas no recomendadas en torno a la interpretación y uso de estos datos de aprendizaje.

La información derivada de esta investigación contribuirá a la generación de nuevos conocimientos sobre la utilización de resultados de aprendizaje. En este caso, en el marco de un contexto educativo contradictorio, pues se insta a los y las docentes a usarlos con fines pedagógicos, pero a la vez, se sienten presionados por demostrar -a través de estos resultados- la eficacia de su desempeño profesional. Para presentar esta investigación, en este artículo se analiza la percepción del profesorado sobre el uso del Informe de Resultados de la prueba SIMCE para determinar si se hace desde una mirada pedagógica (nuevo enfoque de la Agencia de Calidad de la Educación) o solo como un mecanismo de rendición de cuentas.

\section{METODOLOGÍA}

Para llevar a cabo este estudio, se seleccionó el enfoque empírico-analítico, positivista o cuantitativo, empleando un estudio descriptivo con el fin de obtener una descripción precisa sobre un fenómeno educativo particular (Bisquerra, 2014). Asimismo, se escogió la encuesta como técnica de recogida de información.

El "Cuestionario sobre uso del Informe de Resultados Educativos del Sistema de Medición de la Calidad de la Educación (SIMCE)" es un instrumento de recogida de información de elaboración propia que responde a los fines de esta investigación, pues se construyó a partir de la consideración de "cuál es la información exacta" (Bisquerra, 2014, p. 246) que se busca obtener. Presenta la siguiente estructura: primero, se indica su propósito y se pide el consentimiento informado de los destinatarios. Luego, se especifican las instrucciones de cumplimentado y se solicita información relativa a los datos personales y académicos de los y las docentes participantes. Finalmente, se recoge información a través de 37 ítems que se miden en una escala Likert de cinco valores, en la 
que 1 significa "Totalmente en desacuerdo"; 2 , "En desacuerdo"; 3, "Ni de acuerdo ni en desacuerdo"; 4, "De acuerdo"; y 5, "Totalmente de acuerdo".

Este cuestionario fue sometido a una validez de contenido a través de un "juicio de expertos" (Escobar-Pérez y Cuervo-Martínez, 2008), cuyo proceso consiste en obtener una opinión de "personas con trayectoria en el tema, que son reconocidas por otros como expertos cualificados en éste, y que pueden dar información, evidencia, juicios y valoraciones" (Escobar-Pérez y CuervoMartínez, 2008, p. 29).

Para llevar a cabo el proceso, tras la construcción de este cuestionario, se elaboró un instrumento de validación a partir de una adaptación de la propuesta de Escobar-Pérez y CuervoMartínez (2008). En su aplicación, se solicitó a los y las especialistas que emitieran una valoración para los criterios de pertinencia y claridad, utilizando para ello una escala del 1 a 4 (1: Nada; 2: Poco; 3: Bastante; 4: Mucho). Si la respuesta era inferior a 4, se les pidió que indicaran una propuesta de mejora destinada a perfeccionar el ítem. Asimismo, tal como lo señalan Escobar-Pérez y CuervoMartínez (2008), se proporcionó a los expertos y las expertas información sobre la finalidad de este cuestionario, con el fin de ofrecer una contextualización que les permitiera emitir un juicio de manera informada.

Posteriormente, a través de la gestión de la directora del centro educacional, se invitó a participar en esta investigación al profesorado directamente implicado en la aplicación de la prueba SIMCE durante el año 2018. Esto es relevante, dado que el Informe de Resultados Educativos invita a hacer una valoración de la metodología de enseñanza empleada durante ese año, con el fin de reflexionar sobre su efectividad y, con ello, tomar decisiones pedagógicas proyectadas a futuro. Por otra parte, se informó detalladamente en qué consistía este estudio y se consideraron los aspectos éticos involucrados, de tal forma que se obtuvo el consentimiento informado de los y las participantes.

Los datos recogidos fueron analizados a través del programa informático SPSS versión 24 . Para determinar la validez de contenido del cuestionario utilizado en esta investigación, se calculó la concordancia entre jueces utilizando el estadístico de Kendall. Asimismo, se optó por la utilización de los estadísticos descriptivos media, desviación estándar y cuartiles, con el fin de conocer la percepción docente de cada una de las dimensiones del cuestionario.

\section{Muestra}

Para llevar a cabo este estudio, se seleccionó un centro de educación primaria y secundaria de dependencia municipal ubicado en Santiago de Chile, pues se trata de una institución cuyo funcionamiento está determinado en gran parte por los resultados de esta prueba estandarizada. Además, se consideró su calidad de "emblemático", dado que en este tipo de centros está muy arraigada la cultura de rendición de cuentas y, en consecuencia, se otorga una gran importancia a la prueba SIMCE (Quaresma y Orellana, 2016). Asimismo, se decidió consultar al profesorado que impartió clases en los niveles evaluados por esta prueba durante el año 2018, pues este Informe de 
Resultados Educativos era el más actual publicado y disponible en la página oficial de la Agencia de Calidad de la Educación durante la realización de este estudio.

De acuerdo con la información otorgada por la Jefa de la Unidad Técnica Pedagógica del centro, la población está compuesta por un total de 61 docentes, quienes ejercen en tres modalidades de enseñanza: Educación Parvularia, Educación Básica y Educación Media, con 5, 14 y 42 integrantes respectivamente. En relación con su organización, el profesorado de Educación Media se agrupa por Departamentos de acuerdo con las distintas áreas del currículo educativo.

Es necesario señalar que no fue posible contactar a todos los y las docentes que ejercieron durante el año 2018, pues -dada la enorme movilidad laboral del sistema educativo chileno- algunos de ellos ya no están impartiendo clases en este centro. Asimismo, por causa de la emergencia sanitaria acaecida por la COVID-19, las clases presenciales se suspendieron en Chile, lo que imposibilitó reunir a todo el profesorado implicado. Estas circunstancias impidieron la obtención de la muestra completa según lo proyectado, por lo que se contó con 17 cuestionarios para este estudio, de un número estimado de 30 docentes.

\section{RESULTADOS Y DISCUSIÓN}

De acuerdo con Parra y Matus (2016), las creencias que tienen los actores educativos determinan la interpretación y el uso que realizarán de los datos de aprendizaje del estudiantado. Por ello, uno de los objetivos que se ha planteado para este estudio es conocer la valoración que los y las docentes tienen de la prueba SIMCE y sus efectos educativos, lo que se puede apreciar en la siguiente tabla:

\section{Tabla 1}

Valoración sobre la función del SIMCE.

\begin{tabular}{|c|c|c|c|c|c|}
\hline & Media & $\begin{array}{l}\text { Desviación } \\
\text { estándar }\end{array}$ & $Q_{1}$ & $\mathrm{Q}_{2}$ & $\mathrm{Q}_{3}$ \\
\hline $\begin{array}{l}\text { Ayuda a las familias a elegir un centro } \\
\text { educativo para sus hijos e hijas. }\end{array}$ & 3,47 & ,943 & 3,00 & 4,00 & 4,00 \\
\hline \multicolumn{6}{|l|}{ Contextualiza los logros y necesidades de } \\
\hline $\begin{array}{l}\text { los estudiantes en el contexto social del } \\
\text { centro. }\end{array}$ & 2,41 & ,795 & 2,00 & 2,00 & 3,00 \\
\hline $\begin{array}{l}\text { Sirve para conocer el nivel de aprendizaje } \\
\text { real de los estudiantes, es decir, si }\end{array}$ & & & & & \\
\hline $\begin{array}{l}\text { efectivamente alcanzaron los objetivos de } \\
\text { aprendizaje propuestos durante el año } \\
\text { académico. }\end{array}$ & 3,12 & 1,166 & 2,00 & 3,00 & 4,00 \\
\hline $\begin{array}{l}\text { Se utiliza para incentivar y/o premiar el } \\
\text { buen desempeño docente. }\end{array}$ & 3,00 & 1,173 & 2,00 & 3,00 & 4,00 \\
\hline
\end{tabular}


Se utiliza para generar acciones que mejoren el aprendizaje de los estudiantes.

Para efectos de esta investigación es relevante conocer la percepción docente sobre uno de los objetivos fundacionales de la implementación de la prueba SIMCE en 1988: ofrecer información que permita a los padres elegir el mejor centro escolar para sus hijos (Botella y Ortiz, 2018). En torno a esta función orientadora, los datos de la presente investigación (tabla 1) señalan que la publicación del Informe de Resultados Educativos permite la consecución de este objetivo, por lo que se observa una coincidencia entre uno de los propósitos originales de su creación y la percepción del profesorado consultado en esta investigación. En lo referente a la interacción pedagógica, el profesorado consultado considera que los resultados del SIMCE se utilizan para generar acciones que mejoran el aprendizaje de los y las estudiantes.

Como un aspecto negativo, los y las docentes observan una falta de contextualización de los logros y necesidades de los y las estudiantes en el contexto social del centro. Esta opinión coincide con una de las deficiencias del SIMCE diagnosticada por el Ministerio de Educación de Chile (2015): la reducción de la noción de calidad educativa, pues esta debería atender al desarrollo integral del estudiantado, además de garantizar la equidad (Agencia de Calidad de la Educación, 2018a).

Sobre la utilidad de esta prueba para conocer la calidad del centro y el nivel de aprendizaje de los y las estudiantes, se observa la falta de un posicionamiento uniforme en torno a su eficacia para medir el aprendizaje que efectivamente han adquirido (tabla 1), pilar fundamental de la calidad educativa (OREALC/UNESCO, 2013).

En cuanto a las creencias de los y las docentes sobre los usos de los datos de aprendizaje de la prueba SIMCE, Botella y Ortiz (2018) señalan que muchos municipios aplican sus propias recompensas o sanciones para el profesorado a partir de los resultados de esta evaluación, práctica estrechamente relacionada con la rendición de cuentas (Pino et al., 2016). Al respecto, la percepción de los y las participantes (tabla 1) sobre la utilización de los resultados en el incentivo y/o premio del desempeño docente presentó una gran dispersión de respuestas, lo que evidencia diferentes posturas al respecto. Esto podría explicarse por los antecedentes sociodemográficos del profesorado de este centro, puesto que en esta institución conviven docentes con escasos años de ejercicio laboral junto con otros con amplia trayectoria en el sistema, por lo que habría una variedad de experiencias con respecto a este punto.

En relación con los usos que los y las docentes hacen de los resultados de evaluaciones de aprendizaje SIMCE, los resultados son los siguientes: 


\section{Tabla 2}

Valoración sobre prácticas recomendadas.

\begin{tabular}{|c|c|c|c|c|c|}
\hline & Media & $\begin{array}{l}\text { Desviación } \\
\text { estándar }\end{array}$ & $\mathrm{Q}_{1}$ & $\mathrm{Q}_{2}$ & $\mathrm{Q}_{3}$ \\
\hline $\begin{array}{l}\text { Evalúa si algún grupo requiere más apoyo o } \\
\text { estrategias distintas a las utilizadas hasta el } \\
\text { momento. }\end{array}$ & 4,12 & ,993 & 4,00 & 4,00 & 5,00 \\
\hline $\begin{array}{l}\text { Autoevalúa las prácticas pedagógicas } \\
\text { desarrolladas en la enseñanza de los resultados } \\
\text { de aprendizaje por eje, con el propósito de } \\
\text { retroalimentar la gestión pedagógica. }\end{array}$ & 4,29 & 985 & 4,00 & 4,00 & 5,00 \\
\hline $\begin{array}{l}\text { Reflexiona sobre los diversos factores } \\
\text { (metodología empleada, tiempo asignado, etc.) } \\
\text { que pueden influir en que existan diferencias en } \\
\text { los logros de aprendizaje de cada eje. }\end{array}$ & 4,18 & ,951 & 4,00 & 4,00 & 5,00 \\
\hline $\begin{array}{l}\text { Compara los resultados entre años para observar } \\
\text { la trayectoria del centro. }\end{array}$ & 3,94 & 1,249 & 4,00 & 4,00 & 5,00 \\
\hline $\begin{array}{l}\text { Compara los resultados en las evaluaciones de } \\
\text { aprendizaje SIMCE con los resultados de otros } \\
\text { centros de similares condiciones } \\
\text { socioeconómicas. }\end{array}$ & 4,00 & 1,118 & 3,00 & 4,00 & 5,00 \\
\hline $\begin{array}{l}\text { Analiza la gestión institucional y pedagógica para } \\
\text { la mejora de los aprendizajes, considerando las } \\
\text { características propias del centro y la experiencia } \\
\text { de otros centros similares. }\end{array}$ & 3,94 & ,899 & 4,00 & 4,00 & 4,00 \\
\hline
\end{tabular}

Como se puede apreciar en la Tabla 2, los y las docentes declararon usar estos resultados para los siguientes fines pedagógicos: evaluar si algún grupo requiere más apoyo o estrategias distintas a las utilizadas hasta el momento; autoevaluar las prácticas pedagógicas desarrolladas en la enseñanza de los resultados de aprendizaje por eje, con el propósito de retroalimentar la gestión pedagógica; analizar la gestión institucional y pedagógica para la mejora de los aprendizajes, considerando las características propias del centro y reflexionar sobre los diversos factores que pueden influir en que existan diferencias en los logros de aprendizaje de cada eje. Se observa, por lo tanto, un lineamiento coherente en torno a la interpretación y uso de la información para la mejora de los aprendizajes del estudiantado, siguiendo las orientaciones explícitas de la Agencia de Calidad de la Educación en el Informe de Resultados Educativos 2018. En términos específicos, el profesorado sigue la línea de acción sugerida por este organismo: identificar desafíos o problemas para abordarlos con datos, tomar decisiones e implementarlas y evaluar la toma de decisiones. Asimismo, existe una visión coincidente con la investigación llevada a cabo por la Agencia de Calidad de la Educación 
(2018b, p. 27) que evidencia la valoración que los y las docentes tienen de la presentación desglosada del Informe de Resultados Educativos, pues les permite "contar con información relevante a nivel pedagógico para el desarrollo de sus estudiantes en estas áreas."

Por otra parte, en cuanto a una de las utilidades de las pruebas estandarizadas, CamposMartínez y Guerrero (2016) afirman que estas permiten emitir juicios sobre el avance o retroceso de la calidad de la educación en las escuelas, así como también posibilitan la identificación de casos excepcionales de centros que logran ofrecer educación de calidad, aún en circunstancias adversas. Desde esta perspectiva, el profesorado consultado en esta investigación (tabla 2) compara los resultados entre años para observar la trayectoria del centro.

En sentido opuesto, sobre las prácticas que la Agencia de Calidad de la Educación recomienda evitar, los resultados son los siguientes:

\section{Tabla 3}

Valoración sobre prácticas que se recomienda evitar.

\begin{tabular}{|c|c|c|c|c|c|}
\hline & Media & $\begin{array}{l}\text { Desviación } \\
\text { estándar }\end{array}$ & $\mathrm{Q}_{1}$ & $Q_{2}$ & $\mathrm{Q}_{3}$ \\
\hline $\begin{array}{l}\text { Evita comparar los resultados entre las distintas } \\
\text { asignaturas evaluadas. }\end{array}$ & 2,59 & ,939 & 2,00 & 2,00 & 3,00 \\
\hline $\begin{array}{l}\text { Evita enfocar los esfuerzos de mejora en el eje que } \\
\text { demuestra tener menos logros de aprendizaje. }\end{array}$ & 2,29 & 1,213 & 1,00 & 2,00 & 3,00 \\
\hline $\begin{array}{l}\text { Evita comparar el resultado del primer año de la } \\
\text { tendencia con el del último año para establecer } \\
\text { diferencias entre ellos. }\end{array}$ & 2,35 & ,931 & 2,00 & 2,00 & 3,00 \\
\hline $\begin{array}{l}\text { Evita relacionar el puntaje por ejes con el puntaje } \\
\text { promedio SIMCE. }\end{array}$ & 2,59 & 870 & 2,00 & 2,00 & 3,00 \\
\hline $\begin{array}{l}\text { Evita atribuir los resultados de aprendizaje a } \\
\text { factores externos que trasciende al trabajo en el } \\
\text { aula y/o en el centro (ambiente familiar, nivel } \\
\text { educacional de los padres, etc.) }\end{array}$ & 2,65 & 996 & 2,00 & 3,00 & 3,50 \\
\hline
\end{tabular}

En general, los datos (tabla 3) transitan desde una posición neutral a una que manifiesta la realización de acciones no recomendadas como, por ejemplo, comparar los puntajes entre las distintas asignaturas evaluadas. Esto se vincula con un efecto negativo de la aplicación de la prueba SIMCE ya diagnosticado, pues el profesorado percibe que lo realmente valorado por la comunidad es el resultado por asignatura (Ministerio de Educación de Chile, 2015). Asimismo, además de observar la trayectoria del centro, los y las docentes (tabla 3) también comparan el resultado del primer año con el último, estableciendo una diferencia entre ellos, lo que evidencia una valoración del "producto final" (puntaje). 
Por otra parte, una práctica asociada con la evaluación es la tendencia a privilegiar aquellos contenidos que son evaluados (De la Orden, 2009). Falabella y Opazo (2014) detectaron una reducción de la implementación curricular como efecto no deseado de la aplicación del SIMCE y Ruminot (2017) evidenció una reorientación del tiempo asignado al tratamiento de las materias en función de lo que mide esta prueba. En esta investigación (tabla 3), los y las docentes declararon enfocar o centrar los esfuerzos de mejora en el eje que demuestra tener menos logros de aprendizaje, lo que sugiere la importancia que se otorga a aquellos contenidos contemplados en esta evaluación. Si relacionamos esta práctica desde una perspectiva mundial, Morris y Barroso-Hurtado (2019) señalan que algunos países, para mejorar sus resultados en la prueba PISA, tienden a incrementar el tiempo dedicado a las áreas evaluadas.

\section{CONCLUSIONES}

Pese a la variedad de aproximaciones sobre el concepto de calidad, existe un acuerdo general en cuanto a la relevancia de la interacción pedagógica, por lo que es importante prestar atención a dos actores fundamentales del sistema: docentes y estudiantes (Agencia de Calidad de la Educación, 2018a). Por ello, esta investigación planteó como objetivo general conocer la percepción del profesorado sobre el uso de los resultados del SIMCE en un centro de educación municipal de Santiago de Chile, pues la utilización que los y las docentes hacen de esta información tiene una repercusión directa en el sistema educativo y en la calidad de este.

En torno a las conclusiones obtenidas, el profesorado manifiesta una posición crítica sobre la presentación de los datos de aprendizaje en el Informe de Resultados Educativos, dado que -según su percepción- no se contextualizan en la realidad social de cada centro. Sin embargo, en una postura coherente con la valoración del contexto del cual provienen los y las estudiantes, el profesorado declara utilizar estos factores contextuales en la interpretación y uso de los resultados de aprendizaje para su quehacer pedagógico.

Sobre aquella información relacionada con el proceso de enseñanza y aprendizaje, los resultados de este estudio indican una valoración general del profesorado en torno a los datos pedagógicos contenidos en el Informe de Resultados Educativos del SIMCE. Esta visión favorable se concreta en su trabajo en el aula, dado que declararon utilizarlos para reflexionar y retroalimentar la efectividad de sus prácticas. Por lo tanto, a partir de esta información, es posible afirmar que los y las docentes participantes en este estudio utilizan estos resultados en concordancia con el foco pedagógico recomendado por la Agencia de Calidad de la Educación, pues los emplean para mejorar el aprendizaje del estudiantado.

Sin embargo, es posible constatar la coexistencia de estas buenas prácticas con aquellas no recomendadas por la Agencia de Calidad de la Educación. Es relevante precisar que estas acciones están vinculadas con la concepción del SIMCE como mecanismo de rendición de cuentas, puesto que el centro siente la presión por demostrar su calidad a través de esta evaluación, razón por la que priman los resultados por sobre el proceso de aprendizaje. En efecto, se observa la comparación de 
resultados entre asignaturas, entre los puntajes del primer año con el último y el enfoque central en los contenidos evaluados en esta prueba. Esto permitiría concluir que el foco pedagógico impulsado por la Agencia no ha logrado desplazar la atención excesiva en la rendición de cuentas.

Este fenómeno se relaciona con el siguiente nudo crítico detectado por Flórez y Oyarzún (2016) en el plan de evaluaciones 2016 - 2020 del SIMCE: "Persiste la tensión por la multiplicidad de objetivos del sistema de medición, donde conviven contradictoriamente dinámicas de presión y apoyo a las escuelas, u objetivos de control estatal con los de retroalimentación pedagógica a partir del mismo instrumento." (p. 8). En esta misma línea, Acuña, Mendoza y Rozas (2019) sostienen que, desde que se ha tomado la decisión de no modificar los cimientos del sistema heredado, todo depende de "la buena o mala voluntad de quien usa el SIMCE." (p. 64)

En consecuencia, esta dicotomía, en la que coexiste un foco pedagógico junto con la presión por rendir cuentas, podría explicar los resultados de esta investigación. Sin embargo, para determinarlo con certeza, es necesario realizar una mayor cantidad de estudios. Para ello, se debería tener como punto de partida la dificultad para perder de vista el foco en el accountability en el contexto de una cultura de la evaluación que por años ha privilegiado la obtención de resultados.

Sobre las implicaciones socioeducativas de esta investigación, es imprescindible tener presente una serie de limitaciones que impiden generalizar sus conclusiones. Entre estos factores, es posible identificar la escasa cantidad de docentes que conformaron la muestra y el contexto excepcional en el que se realizó el estudio debido a la emergencia sanitaria mundial causada por la COVID-19. Pese a todo, es importante considerar que aún no existen investigaciones publicadas sobre la alineación del profesorado a las nuevas recomendaciones de la Agencia de Calidad de la Educación con respecto al uso del Informe de Resultados Educativos del SIMCE. Desde esta perspectiva, este estudio podría contribuir a realizar un primer acercamiento a una realidad educativa, proporcionando "datos que vayan preparando el camino para nuevas investigaciones" (Bisquerra, 2014, p. 233).

En este sentido, esta investigación podría constituir un aporte para futuros estudios destinados a indagar y profundizar en este fenómeno como, por ejemplo: ampliar la muestra a docentes que se desempeñen en centros de distintas dependencias, tales como municipal, privada o particular subvencionado, con el fin de averiguar si esta variable influye en los resultados. $Y$ esta generación de conocimientos podría utilizarse para retroalimentar el proceso de aplicación de esta prueba estandarizada, la que -sin duda- ha tenido y tiene un gran impacto en el sistema educativo de Chile.

\section{REFERENCIAS}

Acuña, F., Mendoza, M., y Rozas, T. (2019). El hechizo del SIMCE: sobre cómo ocultar la competencia escolar en nombre del aseguramiento de la calidad de la educación. Revista Chilena de Pedagogía, 1(1), 54-70. DOI 10.5354/2452-5855.2019.55633 
Agencia de Calidad de la Educación. (2018a). Tarea de todos. Hacia una visión compartida de la calidad de la educación. http://archivos.agenciaeducacion.cl/060308_TAREADETODOS_ONLINE.pdf

Agencia de Calidad de la Educación. (2018b). Nuevo Sistema Nacional de Evaluación de Aprendizajes. La evaluación al servicio de los aprendizajes. http://archivos.agenciaeducacion.cl/Sistema_Nacional_de_Evaluacion_17abr.pdf

Anghel, B., Cabrales, A., Sainz, J., y Sanz, I. (2013). Publicación de los resultados de las pruebas estandarizadas externas: ¿Tiene ello un efecto sobre los resultados escolares? En A. Cabrales y A. Ciccone (coords.), La educación en España. Una visión académica (pp. 16-47). Fedea.

Aristizabal, J. A. (2016). Analítica de datos de aprendizaje (ADA) y gestión educativa. Revista Gestión de la Educación, 6(2), 149-168. https://doi.org/10.15517/rge.v1i2.25499

Ávila-Gómez, M. (2016). La evaluación de calidad como medio de transformación educativa. Revista Educación, Política y Sociedad, 1(1), 50-69.

Backhoff, E. (2018). Evaluación estandarizada del logro educativo: contribuciones y retos. RDU $\begin{array}{llll}\text { Revista Digital } & \text { Universitaria, } & 19(6), & \text { 1-14. }\end{array}$ http://doi.org/10.22201/codeic.16076079e.2018.v19n6.a3

Bisquerra, R. (coord.). (2014). Metodología de la investigación educativa. La Muralla.

Bordoli, E. y Márquez, M. (2019). Evaluaciones internacionales de aprendizaje y posiciones docentes: dislocación y nuevos sentidos. Un análisis crítico. Foro de Educación, 17(26), 25-44. https://doi.org/10.14516/fde.707

Botella, M. T. y Ortiz, C.P. (2018). Efectos indeseados a partir de los resultados SIMCE en Chile. Revista Educación, Política y Sociedad, 3(2), 27-44. http://hdl.handle.net/10486/684909

Campos-Martínez, J. y Guerrero, P. (2016). Efectos indeseados de la medición de la calidad educativa en Chile. La respuesta de la sociedad civil. Cadernos Cedes. 36(100), 335-374. https://doi.org/10.1590/CC0101-32622016171351

Carrasco, A. y Urrejola, A. (2017). La Agencia de Calidad de la Educación, ¿Una política de evaluación integral? Revista Educación, Política y Sociedad, 2(1), 8-26.

De la Orden, A. (2009). Evaluación y calidad: análisis de un modelo. Estudios sobre Educación, 16(1), 17-36.

Díaz, K. y Osuna, C. (2016). Las evaluaciones estandarizadas del aprendizaje y la mejora de la calidad educativa. Temas de educación 22(1), 131-146.

Escobar-Pérez, J. y Cuervo-Martínez, A. (2008). Validez de contenido y juicio de expertos: una aproximación a su utilización. Avances en Medición, 6, 27-36.

Falabella, A. y Opazo, C. (2014). Sistema de Aseguramiento de la Calidad y procesos de mejoramiento: una mirada desde la gestión educativa. United Nations Educational Scientific and Cultural Organization (UNESCO) y Ministerio de Educación de Chile. https://www.researchgate.net/publication/330667110

Flórez, M. T. y Oyarzún, G. (2016). Resultados SIMCE y Plan de Evaluaciones 2016-2020: Nudos críticos y perspectivas de cambio. Cuaderno de Educación,73, 2-11. 
García-Sanz, M. P. (2012). Fundamentos teóricos y metodológicos de la evaluación de programas. DM.

Ministerio de Educación de Chile. (2015). Hacia un sistema completo y equilibrado de evaluación de los aprendizajes en Chile. Informe Equipo de Tarea para la Revisión del SIMCE. https://www.mineduc.cl/2015/11/04/informe-del-equipo-de-tarea-para-la-revision-del-simcehacia-un-sistema-completo-y-equilibrado-de-evaluacion-de-los-aprendizajes-en-chile/

Moreno, T. (2011). La cultura de la evaluación y la mejora de la escuela. Perfiles educativos, 33(131), 116-130.

Moreno, T. (2016). Las pruebas estandarizadas en la escuela contemporánea, ¿llave o cerrojo para la mejora de la educación? Temas de Educación, 22(1), 83-96. https://revistas.userena.cl/index.php/teduacion/article/view/738

Moreno, T. (2017). La evaluación, ¿nos conducirá a la tierra prometida? Perspectiva Educacional. Formación de Profesores, 56(1), 147-163. https://doi.org/10.4151/07189729

Morris, P. y Barroso-Hurtado, D. (2019). Pruebas internacionales y la búsqueda de la escolarización de "clase mundial": un análisis crítico de narrativas políticas. Foro de Educación, 17(26), 4572. https://doi.org/10.14516/fde.706

Oficina Regional de Educación de la UNESCO para América Latina y el Caribe. (2013). Situación Educativa de América Latina y El Caribe: hacia la educación de calidad para todos al 2015. http://www.unesco.org/new/fileadmin/MULTIMEDIA/FIELD/Santiago/images/SITIEDespanol.pdf

Organización de las Naciones Unidas para la Educación, la Ciencia y la Cultura. (2017). Evaluación del aprendizaje en la UNESCO. Garantía de un aprendizaje efectivo y relevante para todas las personas. https://unesdoc.unesco.org/ark:/48223/pf0000260325_spa

Parra, V. y Matus, G. (2016). Usos de datos y mejora escolar: Una aproximación a los sentidos y prácticas educativas subyacentes a los procesos de toma de decisiones. Calidad en la Educación. 45, 207-250. https://doi.org/10.4067/S0718-45652016000200007

Pino, M., Oyarzún, G., y Salinas, I. (2016). Crítica a la rendición de cuentas: narrativa de resistencia al sistema de evaluación en Chile. Cadernos Cedes. 36(100), 337-354. https://doi.org/10.1590/CC0101-32622016171362

Quaresma, M. L. y Orellana, V. (2016). El accountability y su impacto en la labor docente: percepciones de los profesores de liceos públicos de alto rendimiento académico en Chile. Currículo sem Fronteiras, 16(2), 316-338.

Rappoport, S. y Sandoval, M. (2015). Inclusión educativa y pruebas estandarizadas de rendimiento. Revista nacional e internacional de educación inclusiva 8(2), 18-29.

Ruminot, C. (2017). Los Efectos Adversos de una Evaluación Nacional sobre las Prácticas de Enseñanza de las Matemáticas: El Caso de SIMCE en Chile. Revista Iberoamericana de Evaluación Educativa, 10(1), 69-87. https://doi.org/10.15366/riee2017.10.1.004 